\title{
Enhancing Intelligent Anemia Detection via Unifying Global and Local Views of Conjunctiva Image with Two-Branch Neural Networks
}

\section{Lijuan Zheng}

South China University of Technology

Shaopeng Liu

Guangdong Polytechnic Normal University

Senping Tian

South China University of Technology

Jianhua Guo

Guangdong Polytechnic Normal University

Xinpeng Wang

Guangdong Polytechnic Normal University

Xiuxiu Liao

Guangdong Polytechnic Normal University

Jiaming Hong ( $\nabla$ hjm@gzucm.edu.cn )

Guangzhou University of Chinese Medicine

\section{Research Article}

Keywords: Intelligent Anemia Detection, Multi-View Learning, Two-Branch Neural Networks, Conjunctiva Image, Anemia

Posted Date: January 3rd, 2022

DOI: https://doi.org/10.21203/rs.3.rs-1170958/v1

License: (c) (1) This work is licensed under a Creative Commons Attribution 4.0 International License. Read Full License 


\section{Enhancing Intelligent Anemia Detection via Unifying Global and Local Views of Conjunctiva Image with Two-Branch Neural Networks}

Lijuan Zheng ${ }^{1}$, Shaopeng Liü,*, Senping Tian', Jianhua $\mathrm{Guo}^{2}$, Xinpeng Wang ${ }^{2}, \mathrm{Xiuxiu} \mathrm{Liao}^{2}$ and Jiaming Hong ${ }^{3, *}$

${ }^{*}$ Corresponding author:

Shaopeng Liu

149265005@qq.com

Jiaming Hong,

hjm@gzucm.edu.cn

${ }^{2}$ Department of Computer

Science, Guangdong

Polytechnic Normal

University, Guangzhou,

China

${ }^{3}$ School of Medical

Information Engineering,

Guangzhou University of

Chinese Medicine,

Guangzhou, China

Full list of author

information is available at the end of the article.

\begin{abstract}
Background: Anemia is one of the most widespread clinical symptoms all over the world, which could bring adverse effects on people's daily life and work. Considering the universality of anemia detection and the inconvenience of traditional blood testing methods, many deep learning detection methods based on image recognition have been developed in recent years, including the methods of anemia detection with individuals' images of conjunctiva. However, existing methods using one single conjunctiva image could not reach comparable accuracy in anemia detection in many real-world application scenarios.

Results: To enhance intelligent anemia detection using conjunctiva images, we proposed a new algorithmic framework which could make full use of the data information contained in the image. To be concrete, we proposed to fully explore the global and local information in the image, and adopted a twobranch neural network architecture to unify the information of these two aspects.
\end{abstract}

Conclusions: Compared with the existing methods, our method can fully explore the information contained in a single conjunctiva image and achieve more reliable anemia detection effect. Compared with other existing methods, the experimental results verified the effectiveness of the new algorithm.

Keywords: Intelligent Anemia Detection; Multi-View Learning; Two-Branch Neural Networks; Conjunctiva Image; Anemia

Background

3 Anemia is a typical clinical symptom that the capacity of red blood cells in human peripheral

blood is lower than the normal range. As one of the most widespread clinical symptoms all over

5 the world, anemia could bring adverse effects on people's daily life and work [1-2]. Therefore,

timely diagnosis and treatment is particularly important for anemia. However, the traditional

diagnosis of anemia requires blood sampling for test with professional equipment, which requires 
extra cost and intervention of professionals, and brings obvious limitation to its wide application in daily life [2-4].

In recent years, in order to improve the convenience of anemia examination, many studies have been carried out to investigate the effective approaches of detecting anemia in a noninvasive manner [4-7]. One of the most promising directions was to deploy artificial intelligent algorithms to discover the correlation of anemia with images of human surface organs, including fingernails, retinas, conjunctivas, etc. In other words, instead of testing blood samples, artificial intelligent algorithms for image classification and recognition are applied in anemia diagnosis [8-9]. Due to their non-invasive manner and non-requirement of professional blood testing, such approaches are less expensive, and seem much attractive than the traditional ones.

In this paper, we focus on developing more effective algorithms for one of such approaches, that is, to detect anemia with photos of conjunctiva [10-12]. Comparing to other similar approaches like diagnosis with retina images, detecting with conjunctiva images requires no extra professional equipment, which brings much convenience to users. Therefore, it can be easily deployed in Web-based platforms like APPs, and people can take photos and upload them at any time to get a diagnosis result immediately.

However, there is some room for improvement in existing algorithms of detecting anemia with conjunctiva images. One of the main issues is that, as was demonstrated in previous reports and in our testing experiments, the existing algorithms could not reach comparable performance with human experts in many real-world applications and could be easily affected by image quality. Such drawbacks might limit its potential application prospects.

To further enhance the performance of anemia diagnosis with the photos of conjunctiva, we 
proposed in this paper a new algorithmic framework to solve such problems. As was discussed in detail later in this paper, the main issue which prevents previous algorithms from achieving higher accuracy may lie in that, they cannot fully exploit the information of the image, especially the local information. Inspired by such analysis, we put forward a new perspective which could unify both global and local information of the image data, and proposed a simple yet effective deep convolutional neural network architecture to fuse them, and obtained a much robust and reliable deep learning model for anemia detection using only one single image of an individual's conjunctiva.

\section{Results}

\section{GLUDA Model}

In this article we design a new deep learning paradigm for intelligent anemia prediction using only one single conjunctiva image. Based on our previous results, we find that when observing the conjunctiva image for diagnosis, one of the main inspirations is that, two different views of information (i.e., the global view and the local view) are simultaneously extracted from one single conjunctiva image, and these two views of information are unified in a reasonable approach to help forming comprehensive diagnosis. Motivated by such observation, we propose that similar operations can be deployed into the deep learning architecture to enhance the effectiveness of the algorithm in anemia detection. Thus, we readjust the basic structure of the classic convolutional neural network model and add the structure of multi-view generation and multi-view data fusion to achieve this goal. The overall framework of the new model is depicted in Fig. 1. We refer the new model as GLUDA model (which is short for the full name: Global and Local view Unifying 


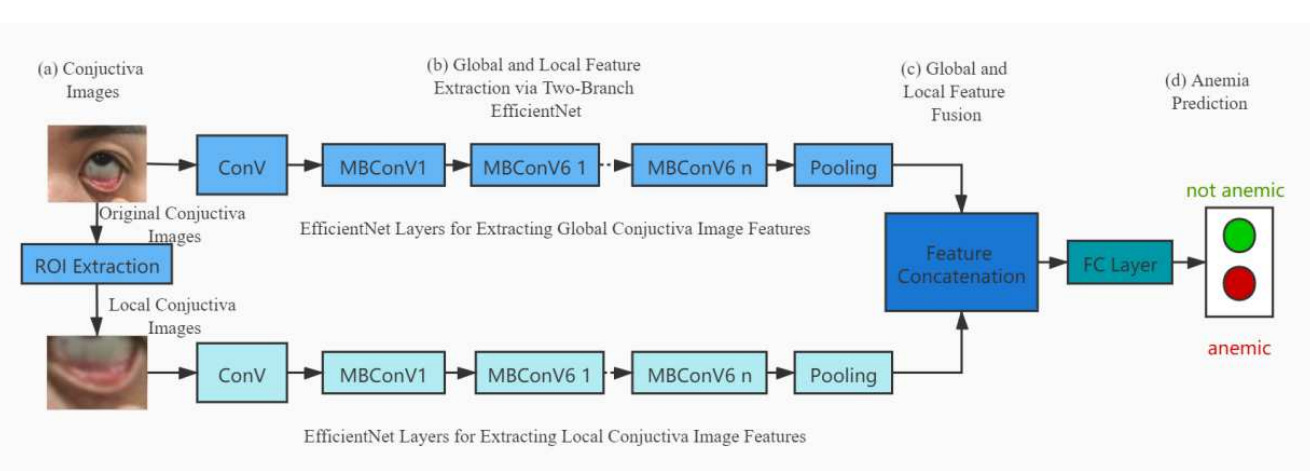

Fig.1 Overall Framework of GLUDA Model

\section{Training framework of the GLUDA}

The GLUDA model benefits from several key aspects, among which the two-branch architecture plays a central role in unifying both global and local information of a single conjunctiva image.

We choose EfficientNet as the two sub-network structure for constructing the whole network.

Recently proposed by Google Brain Team, EffiecientNet is an efficient convolutional neural network, which can scale up CNN models by balancing network depth, width, and resolution in a systemic way. Formally, denote the input data image as an input tensor $\mathrm{X}$, then the output of EfficientNet can be formally defined as:

$$
\operatorname{Eff}(\mathrm{X})=F_{K}^{d} \odot F_{K-1}^{d} \cdots \odot F_{2}^{d} \odot F_{1}^{d}(X),
$$

where each $F_{i}^{d}$ is the ith layer of the network, for $\mathrm{i}=1,2, \ldots, \mathrm{K}$, with $\mathrm{K}$ the total number of all the convolutional layers of the sub-network, and d the coefficients for scaling network depth. To get a more compact expression, we can further denote the ith input tensor as $X_{\left\langle r \cdot H_{i}, r \cdot W_{i}, w \cdot C_{i}\right\rangle}$, where $<$ $\left.H_{i}, W_{i}, C_{i}\right\rangle$ is predefined tensor shape of the corresponding layer, and $\langle d, r, w\rangle$ is the parameter tuples for scaling network depth, width, and resolution. Therefore, for any input tensor 

to as:

and

$\mathrm{X}$ (as a conjunctiva image), the two sub-network Net $i(\mathrm{i}=1,2)$ in GLUDA can be formally referred

$$
\operatorname{Net1}(\mathrm{X}, \mathrm{d}, \mathrm{w}, \mathrm{r})=\bigodot_{i=1,2, \ldots, S} F_{i}^{d, L_{i}}\left(X_{<r \cdot H_{i}, r \cdot W_{i}, w \cdot C_{i}>}\right)
$$

$$
\operatorname{Net} 2(\operatorname{local}(\mathrm{X}), \mathrm{d}, \mathrm{w}, \mathrm{r})=\bigodot_{i=1,2, \ldots, S} F_{i}^{d, L_{i}}\left(\operatorname{local}(X)_{<r \cdot H_{i}, r \cdot W_{i}, w \cdot C_{i}>}\right)
$$

Note that we would construct the two EfficientNet sub-branches with exactly the same structure, and therefore they would share the same predefined tensor shape $<H_{i}, W_{i}, C_{i}>$ in each layer. But the weight parameters would be different after training. Denote the concatenation operation as $F_{\text {conca }}$, and the fully connected layer as $F_{c}$, the output of the GLUDA network can be written as :

$$
\operatorname{Net}(\mathrm{X}, \mathrm{d}, \mathrm{w}, \mathrm{r})=F_{c} \odot\left(F_{\text {conca }}(\operatorname{Net} 1(X, d, w, r), \operatorname{Net} 2(\operatorname{local}(X), d, w, r))\right) \text {. }
$$

To bring all the formal discussions above, the overall training framework of the GLUDA model can be summarized as follows:

$\operatorname{Max} \operatorname{Accuracy}(\operatorname{Net}(d, w, r))$

$$
\begin{gathered}
\text { s.t. } \\
\operatorname{Net1}(\mathrm{X}, \mathrm{d}, \mathrm{w}, \mathrm{r})=\bigodot_{i=1,2, \ldots, S} F_{i}^{d, L_{i}}\left(X_{<r \cdot H_{i}, r \cdot W_{i}, w \cdot C_{i}>}\right) \\
\operatorname{Net2}(\mathrm{X}, \mathrm{d}, \mathrm{w}, \mathrm{r})=\bigodot_{i=1,2, \ldots, S} F_{i}^{d, L_{i}}\left(X_{<r \cdot H_{i}, r \cdot W_{i}, w \cdot C_{i}>}\right) \\
\operatorname{Net}(\mathrm{X})=F_{c} \odot\left(F_{\text {conca }}(\operatorname{Net} 1(X, d, w, r), \operatorname{Net} 2(\operatorname{local}(X), d, w, r))\right), \\
\operatorname{Memory}(\mathrm{Net}) \leq \text { memory_constraints, } \\
F L O P S(\mathrm{Net}) \leq \text { flops_constraints, }
\end{gathered}
$$

where memory_constraints and flops_constraints are constraints of the computation resources. To obtain the optimal parameters for the whole network, compound scaling method and a small 
grid search could be applied. Briefly speaking, the compound scaling method would set $d=$ $\alpha^{\emptyset}, w=\beta^{\emptyset}, r=\gamma^{\emptyset}$, and $\alpha \cdot \beta^{2} \cdot \gamma^{2} \approx 2$, with user-specific coefficient $\emptyset$ and search optimal $\alpha \geq$ $1, \beta \geq 1, \gamma \geq 1$ for appropriate depth, width and resolution settings for the network.

From the formal optimization target in Eq.(5), and considering the structure of conjunctiva images processed in our empirical experiments, below we show the experiment result of the proposed GLUDA model.

\section{Experiment Results}

To evaluate the proposed model, we collected conjunctiva image data from patients enrolled in Zhongshan Ophahalmic Center, Third affiliated Hospital at Sun Yat-sent University and People's Hospital of Guangxi Zhuang Autonomous Region, from June 2019 to November 2019. To make a more comprehensive comparison, we developed two series of model training and testing in our experiments, considering whether data augmentation operation was incorporated in the learning process.

We reported the experimental results of both series in Table 1, Fig. 2 and Fig.3. Row 2 to row 4 in Table 1 and Fig. 2 showed testing results of models without data augmentation. And row 6 to row 8 in Table 1 and Fig. 3 showed testing results of models using data augmentation, where random rotation, cropping and flipping and applied and the size of the augmented training set are extended to 1396 in total. In each series of the experiments, GLUDA models outperformed EfficientNet_Origin and EfficientNet_ROI, respectively. When data augmentation was not adopted, GLUDA model achieved the highest scores among all the three models, with AUC $=$ 0.9087, Acc $=0.7937$, and EfficientNet_ROI obtained the lowest score, with AUC $=$ 0.9305, Acc $=0.8313$. The sensitivity and specificity scores and their corresponding $95 \% \mathrm{CI}$ 


\begin{tabular}{lccc}
\hline Not Augmented & Acc (95\% CI) & Sen (95\% CI) & Spe (95\% CI) \\
\hline GLUDA & $0.7937(0.8539,0.9442)$ & $0.8542(0.7245,0.8492)$ & $0.7679(0.7912,0.9005)$ \\
\hline EfficientNet_Origin & $0.7604(0.7801,0.9179)$ & $0.8056(0.6661,0.8347)$ & $0.7333(0.7153,0.8723)$ \\
\hline EfficientNet_ROI & $0.7125(0.7662,0.8818)$ & $0.8043(0.638,0.777)$ & $0.6754(0.736,0.8584)$ \\
\hline Augmented & Acc (95\% CI) & Sen (95\% CI) & Spe (95\% CI) \\
\hline GLUDA & $0.8313(0.8802,0.9606)$ & $0.8723(0.656,0.8814)$ & $0.8142(0.8118,0.9154)$ \\
\hline EfficientNet_Origin & $0.7812(0.6886,0.8522)$ & $0.8649(0.7823,0.9193)$ & $0.7419(0.6324,0.80779)$ \\
\hline EfficientNet_ROI & $0.75(0.6776,0.8107)$ & $0.7872(0.7175,0.8435)$ & $0.7345(0.6612,0.7969)$ \\
\hline
\end{tabular}

intervals are also the highest for the GLUDA model. When data augmentation was applied before training, we could observe that all three models could achieved higher scores comparing to their counterpart model without augmentation, and among the three models, GLUDA could still obtained the highest scores in AUC, Acc, Sen, and Spe, respectively. According to such comparison results, GLUDA model could fuse global and local views of conjunctiva images in an effective approach, therefore outperformed the other two models which only use global (EfficientNet_Origin) or local (EfficientNet_ROI) views of conjunctiva images.

Table 1. Performance of GLUDA vs. other models in the test set

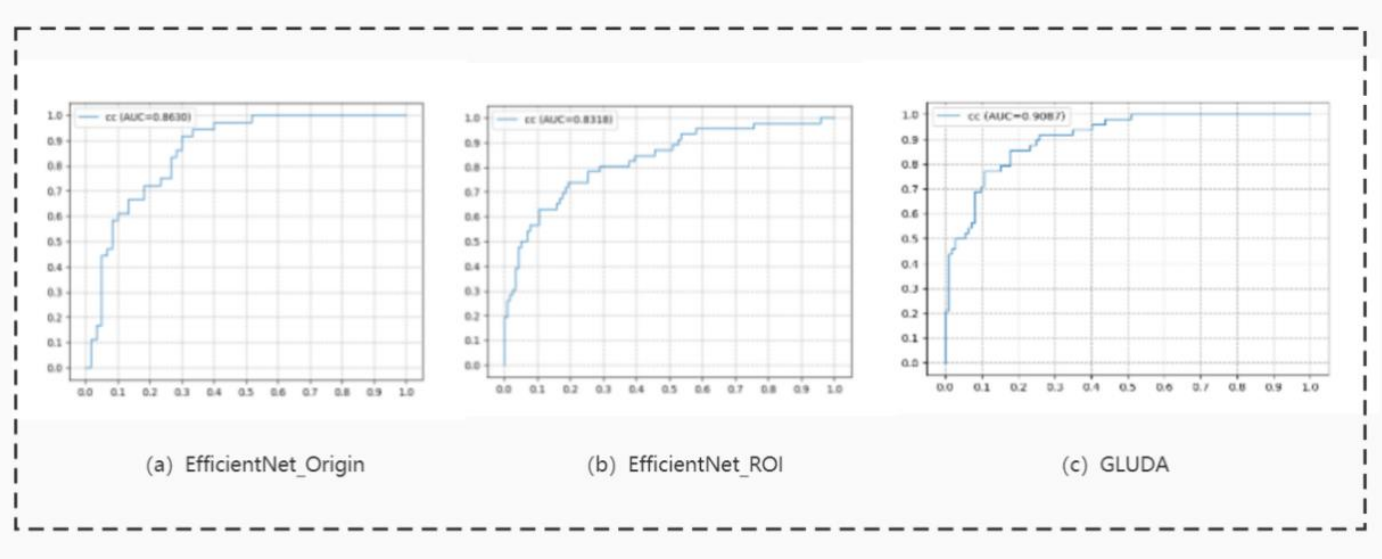

Fig 2. Performance of GLUDA vs. other models without data augmentation 
125

126

127

128

129

130

131

132

133

134

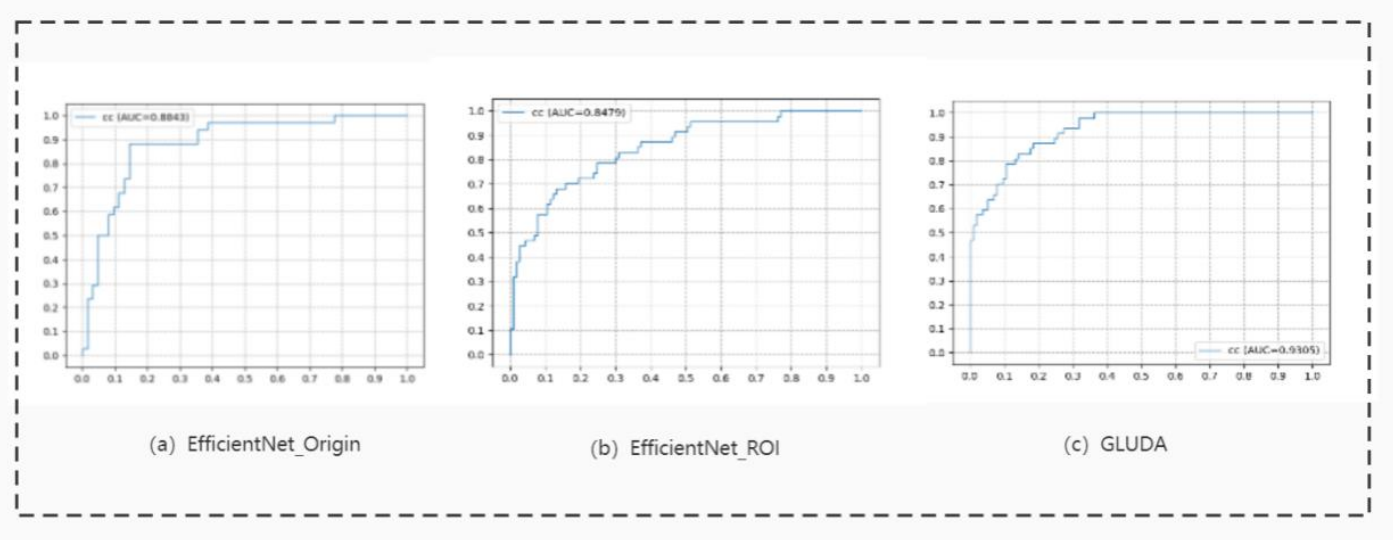

Fig 3. Performance of GLUDA vs. other models with data augmentation

\section{Discussion}

\section{Model discussion}

The heuristic of the new model was motivated by observing how professionals form their comprehensive diagnosis of anemia with the anemia images. Let's consider the following decision-making process: as a professional, when using a conjunctiva image for assisting anemia diagnosis, one would first make a preliminary judgment on the overall image of conjunctiva, considering the potential risk of whether the corresponding individual has anemia. Then, in order to further verify or reject this judgment, one will continue to carefully observe the regions of interest (ROI), and make comparative analysis with the whole image, and finally get a comprehensive judgment of the anemia diagnosis.

Several key steps in the GLUDA model can be summarized as follows:

Firstly, in addition to the original image data serving as the global view, the region of interest (ROI) from the original image, serving as the local view data. Formally, for any conjunctiva image data $x_{s}$, we perform ROI extraction algorithm to produce the local view data, denoted as:

$$
x_{s}^{\prime}=\operatorname{local}\left(x_{s}\right)
$$


143

is then resized to the same size of $x_{s}$, which is also denoted as $x_{s}^{\prime}$ for convenience. $x_{s}$ and $x_{s}^{\prime}$ is then treated as two equal parts to form a tuple as the input of the model, i.e.,

$$
x_{s} \rightarrow\left(x_{s}, x_{s}^{\prime}\right)
$$

and the learning task is reframed to obtain a mapping function $f$ which produce the following mapping for any $\left(x_{s}, y_{s}\right)$ with high generalization ability:

$$
f:\left(x_{s}, x_{s}^{\prime}\right) \rightarrow y_{s} .
$$

Secondly, the overall framework comprises two branches of deep convolutional neural networks, which server as feature extractor for the original conjunctiva image and the ROI conjunctiva image, respectively. The network block architecture of each branch is identical to that of the EfficientNet model, which comprises convolutional layers, mobile inverted bottleneck MBConv layers and Pooling layers as its building blocks [25-27]. There are various versions of EfficientNet model, and we adopt EfficientNet-B0 in our study for convenience but note that other versions can substitute for EfficientNet-B0 here without substantial influence.

Thirdly, the extracted higher-level features of the two branches are fused together, and are passed through fully connected layers to learn a more comprehensive prediction. To be concrete, for each view of the image data, after passing through the layers of the branch, a set of higherlevel features are obtained. To fuse these two sets of features, a simple concatenation operation was carried out, which would concatenate these two sources of features into a unified one. The fused features are then eventually passed through the FC layer to output a prediction of anemia. Note that alternative feature fusion operations other than concatenation might also be applicable here, but simple concatenation operation has well served the task here and works well in practice. 
One of the aspects worth emphasizing is that, we could observe from the results that, even when training without data augmentation, the obtained GLUDA model could still outperformed results in Fig.4. Such contrast is impressive, considering that in this case GLUDA only used 532 conjunctiva images for training, which is far less than the 1396 augmented images for training EfficientNet_Origin and EfficientNet_ROI. Given that data augmentation could provide extra information to boost model training in many cases, it might be reasonable to conclude that the way GLUDA fuse two views of conjunctiva images had successfully draw into valid discriminative information for training, which would be missing when only a single view of conjunctiva images was used. Such augmentation of information is parallel to that of data augmentation, since the performance of GLUDA could be further improved when trained with augmented set, which could be found in Figure 4.

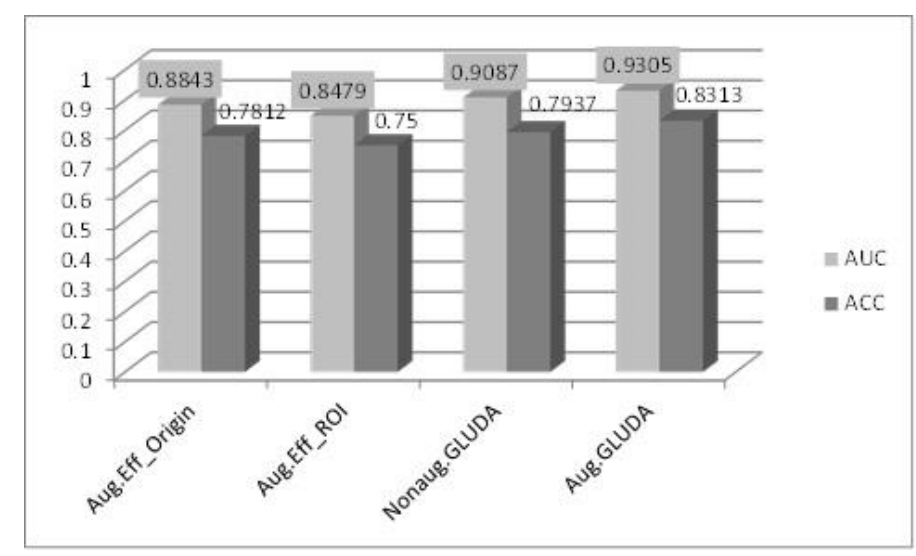

\section{Conclusion}


In this paper, we proposed a new algorithmic framework for the problem of anemia detection using individuals' images of conjunctiva. Compared with the traditional blood testing method, this method has great advantages in the convenience of application due to its inventiveness manner. However, existing work using one single conjunctiva image could not reach expected accuracy in many real-world application scenarios. Motivated by the observation that professionals can benefit from their abilities in comprehensively unifying both global and local information of a single conjunctiva image in enhancing the accuracy of anemia diagnosis, we proposed a two-branch neural network architecture to fully explore such two views of the conjunctiva image and achieved a much robust and reliable performance in intelligent diagnosis.

In our future work, we would continue to investigate other alternative approaches of unifying multiple views of conjunctiva image data other than the proposed two-branch neural network approach. Furthermore, considering that the paradigm proposed might benefit other clinical or medical application tasks involving image recognition, we will continue to explore more potential applications of this method.

\section{Methods}

\section{Various approaches in detecting anemia}

Here, we review some of the related work, including the various approaches in detecting anemia with images, and one of the main deep learning research in processing multi-modal data, i.e., multi-modal deep learning, whose idea was adopted in designing the proposed new algorithm.

Anemia detection methods without blood testing: Due to the universality of anemia detection and the inconvenience of traditional blood detection methods, several alternative non-invasive 
approaches have been investigated in recent years. For instance, several previous works have been done to investigate the correlation of anemia and physical signs such as the pallor of conjunctivae, tongue, palms and nailbed $[7,12,13]$. Since such approaches often need human intervention for estimation and are prone to be affected by subjective judgement of people, they have the disadvantages of instability and labor-intensive.

In order to enhance the objectivity and stability of diagnosis, more methods based on automatic image processing and recognition have been developed. For example, [9] proposed to use multilinear regression to estimates hemoglobin levels with color data and metadata of fingernail bed smartphone photos, and use them as indicated index for detecting anemia. [10] proposed to use neural networks for building detecting models with conjunctiva images. [3] proposed a deep learning algorithm which was trained using retinal images and alternative metadata to output predicts of blood-haemoglobin levels for anemia diagnosis. [15] developed a smartphone App to estimate hemoglobin level for detecting anemia given a light source shining through a patient's finger.

The above approaches mainly rely on traditional image processing technology which is prone to be affected by the image defects in color and other aspects, and often needs experts' intervention to justify the image quality, making them instability and labor-intensive. Therefore, many researchers choose to switch to deep learning approach for more stable and intelligent detection. Such approach aims at training deep learning models with a set of annotated images, which would give automatic diagnosis without any intervention of professionals. [11] Developed a deep learning approach which uses fundus retinal images plus patients' metadata to perform automated anemia screening. [16] constructed neural network models for anemia detection using conjunctiva 
images. [17] proposed a U-Net based conjunctiva segmentation model to detecting anemia. To summarize, previous work of this line have showed the huge potential of deep learning for anemia detection with conjunctiva images, but there is still more room for improvement to make such technology more feasible and robust.

Deep Learning for Multimodality Medical Data: Many practical medical or clinical applications involve multiple modalities rather than single modality data. Therefore, the analysis and processing of multimodality medical data with deep neural network has gradually become a research hotspot [18-19]. Hyperdensenet [20] is a 3D complete convolutional neural network, which extends dense connection to multimodal medical image segmentation task. Experiments show that hyperdensenet achieves satisfactory results in the brain tissue segmentation challenge. In [21], a network suitable for multimodal medical image scene was designed to fuse the complementary anatomical and functional information from different modal images of PET and $\mathrm{CT}$, so as to realize the automatic detection of lung cancer. [22] uses multimodal (B-scan, color Doppler, elastography), multiple angle (cross section and vertical section) breast image data sets to construct an interpretable deep convolution neural network for multi-channel ultrasound image fusion. MMFNet [23] uses multiple encoders to extract multimodal MRI image features, and then fuses the features to complete the semantic segmentation task of nasopharyngeal carcinoma through the decoders. In [24], the novel coronavirus pneumonia severity was predicted with convolution neural network and regression analysis model, using both CT images and structured information from medical records. To the best of our knowledge, little work on multimodality deep learning for anemia detection has been done in previous works, and there is a lot of room for exploration in this research direction. And the approach proposed in this study might be a useful 
exploration in applying multimodal algorithms in unifying both global and local information in this aspect.

\section{Problem formulation}

Formally, the learning task of this study is as follows: given a collection of conjunctiva images as training data instances, with each image labeled with $\mathrm{Y} / \mathrm{N}$ annotated by professionals ( $\mathrm{Y}$ meaning the corresponding individual is diagnosed as having anemia and $\mathrm{N}$ meaning otherwise), the target of the task is to construct a deep learning model, which could output an anemia prediction at professional level for any individual, after given his/her image of conjunctiva. To be concrete, we can formally describe the task as: given training data set $\mathrm{D}=\left\{\left(X_{s}, y_{s}\right), s=1,2, \ldots, m\right\}$, where $X_{s}$ denotes the conjunctiva image of the $s$-th individual, $m$ denotes the sample size, and $y_{s}$ denotes the diagnosis result of anemia status of the corresponding individual. We wish to construct a deep learning model for mapping $X_{s}$ to $y_{s}$ with high accuracy and generalization ability.

As was confirmed by previous clinical research and empirical studies, the rich information contained in conjunctiva images can really provide a sufficient basis for the diagnosis of anemia. Therefore, such learning task is empirically solvable, and all we need to do is to design more effective models based on the characteristics of the data and the task.

However, there is some room for improvement in existing algorithms of in this line of work. To be concrete, as was demonstrated by previous research and in our testing experiments, when using a single conjunctiva image with traditional $\mathrm{CNN}$ architecture for anemia detection, it might be difficult to achieve professional judgment accuracy in many cases, especially when there are problems such as unqualified shooting quality or color difference in the images. 

situation is, the current deep learning model applied to anemia detection does not fully consider correlation of the global and local information of the image, which makes it easy to encounter problems when it encounters images with poor image quality. We believe that the ability to combine global and local information is an important reason why experts can obtain high accuracy diagnosis. To this end, we proceed to design a deep learning structure with this fusion ability.

\section{Data preparation}

All images obtained are anonymized before the experiment, and informed consent was obtained from all the patients. The whole data set contains a total of 852 conjunctiva images, with labels of anemic or non_anemic respectively, and each image's label was obtained according to the corresponding patient's laboratory $\mathrm{Hb}$ measurements within 24 hours of photography. All the images are resized to $224 * 224 * 3$ before training. The whole data was randomly divided into three sub-sets, i.e., training set, verification set and test set. We randomly select around $60 \%$ of the non_anemic images to form the verification set, and the rest of all the images are put together to form the test set. Note that we divided the whole data set in this manner to ensure that the proportion of positive and negative instances remains almost unchanged across the three sub-sets. The information of the data set with size of each class is showed in Table 2 .

\begin{tabular}{cccc}
\hline class label & non_anemic & anemic & total \\
\hline whole data & 575 & 277 & 852 \\
& & & \\
\hline
\end{tabular}




\begin{tabular}{cccc}
\hline training set & 353 & 179 & 532 \\
verification set & 109 & 51 & 160 \\
test set & 113 & 47 & 160 \\
\hline
\end{tabular}

\section{Evaluation criteria}

293 Considering that the sample sizes for different classes are unbalanced in our data set (the sample

294 size of non_anemic conjunctiva images are more than two times of the size of anemic ones), we

295 use focal loss [28] as loss functions for our GLUDA model and other comparing models.

296 Formally, for every conjunctiva image, if the model output the probability of the ground truth 297 label as $p_{t}$, then its focal loss would be:

$$
\operatorname{FL}\left(p_{t}\right)=-\left(1-p_{t}\right)^{\gamma} \log \left(p_{t}\right)
$$

where $\gamma$ is a user-defined balanced parameter. As is universally acknowledged, focal loss function is specially designed for training problems with class imbalance. For all the models trained in this section, we simply set $\gamma$ to be 0.5 across all experiments. written as follows:

$$
\text { Sen }=T P /(T P+F N)
$$

$$
\text { Spe }=T N /(T N+F P)
$$




$$
\mathrm{Acc}=(T P+T N) /(T P+F P+T N+F N)
$$

To evaluation the performance of GLUDA model, especially its effectiveness in unifying both global and local views of conjunctiva images, we choose the state-of-the-art EfficientNet as the base model, and use various forms of conjunctiva images as input to obtain different models for comparison. Since GLUDA model uses building blocks of EfficientNet as its main components, such choice of model is relatively reasonable. Specifically, to verify the effectiveness of GLUDA in unifying both global and local views of conjunctiva images, we benchmarked it with the following two models:

(1) EfficientNet_Origin: EfficientNet model trained using the original conjunctiva images;

(2) EfficientNet_ROI: EfficientNet model trained using the ROI of the original conjunctiva images. described as follows. First, as is pre-processed above, we set the input data format as $224 * 224 * 3$, and use the default network structure of EfficientNet_B0 to form the framework of EfficientNet_Origin, EfficientNet_ROI, and the corresponding building blocks of GLUDA. We adopted transfer learning with the pre-trained EfficientNet_B0 to fine tune the models. We use Adaptive Moment Estimation (Adam) as the optimizer in training all the models, and set the learning rate as 0.003 , the learning rate decay factor as 0.99 and the batch size as 16 , respectively. 
332

333

334

335

336

337

338

339

340

341

342

343

344

345

346

347

348

349

350

351

models (EfficientNet_Origin, EfficientNet_ROI and GLUDA, respectively) on test set are calculated and output for performance comparison.

\section{Declarations}

Ethics approval and consent to participate

This study procedure was approved by the Ethics Committee of Third affiliated Hospital at Sun

Yat-sent University, and written informed consent was acquired from all participants.

\section{Consent for publication}

All participants gave written informed consent for the publication of findings.

\section{Availability of data and materials}

The data sets used and/or analyzed during the current study are available from the corresponding author on reasonable request.

\section{Competing interests}

The authors declare that they have no competing interests.

\section{Funding}

This work was supported by the National Natural Science Foundation of China, [Grant Number 62173151, Lijuan Zheng, Senping Tian], Natural Science Foundation of Guangdong Province [Grant Number 2019A1515012048, Shaopeng Liu, Jianhua Guo], The Opening Project of Guangdong Province Key Laboratory of Big Data Analysis and Processing at Sun Yat-sen University, [Grant Number 202001, Jiaming Hong], The Social Sciences Project of Guangzhou 
353

354

355

356

357

358

359

360

361

362

363

\section{Authors' contributions}

Conception and design: Lijuan Zheng, Shaopeng Liu, and Jiaming Hong. Administrative support:

Senping Tian, Shaopeng Liu and Jiaming Hong ; Collection and assembly of data: Xinpeng Wang ,Xiuxiu Liao and Jianhua Guo, ; Data analysis and interpretation: Jiaming Hong, Shaopeng Liu, Xiuxiu Liao, Lijuan Zheng, and Senping Tian; Manuscript writing: All authors; Final approval of manuscript: All authors.

\section{Acknowledgements}

Not applicable.

\section{Authors' information}

${ }^{1}$ Department of Automation Science and Engineering, South China University of Technology,

Guangzhou, China. ${ }^{2}$ Department of Computer Science, Guangdong Polytechnic Normal

University, Guangzhou, China. ${ }^{3}$ School of Medical Information Engineering, Guangzhou

University of Chinese Medicine, Guangzhou, China.

\section{References}

[1] Smith RE, JR. The clinical and economic burden of anemia. The American journal of managed care (2010)

[2] WHO. Haemoglobin concentrations for the diagnosis of anaemia and assessment of severity: 
WHO; 2011

[3] Tham Y C, Cheng C Y, Wong T Y . Detection of anaemia from retinal images. Nature Biomedical Engineering, 2019, 4(1):1-2.

[4] Kim T , Choi S H , Lambert-Cheatham N, et al. Toward laboratory blood test-comparable photometric assessments for anemia in veterinary hematology[J]. Journal of Biomedical Optics, 2016, 21(10):107001.

[5] Shah N, Osea E A , Martinez G J . Accuracy of noninvasive hemoglobin and invasive point of - care hemoglobin testing compared with a laboratory analyzer[J]. International Journal of Laboratory Hematology, 2014, 36(1)

[6] Wittenmeier E, Bellosevich S, Mauff S, et al. Comparison of the gold standard of hemoglobin measurement with the clinical standard (BGA) and noninvasive hemoglobin measurement $(\mathrm{SpHb})$ in small children: a prospective diagnostic observational study[J]. Paediatr Anaesth, 2015, 25(10):1046-1053.

[7] Ashwini K, Mandar K, Rajnish J, et al. Accuracy and Reliability of Pallor for Detecting Anaemia: A Hospital-Based Diagnostic Accuracy Study[J]. Plos One, 2010, 5(1):e8545.

[8] Mitani A, Huang A, Venugopalan S, et al. Detection of anaemia from retinal fundus images via deep learning[J]. Nature Biomedical Engineering, 2020, 4(Suppl.):1-10.

[9] Mannino R G , Myers D R , Tyburski E A, et al. Smartphone app for non-invasive detection of anemia using only patient-sourced photos[J]. Nature Communications, 2018, 9(1).

[10] Collings S, Thompson O, Hirst E, et al. Non-Invasive Detection of Anaemia Using Digital Photographs of the Conjunctiva[J]. Plos One, 2016, 11(4):e0153286.

[11] Jain P , Bauskar S , Gyanchandani M . Neural network based non - invasive method to detect 
394

395

anemia from images of eye conjunctiva[J]. International Journal of Imaging Systems and Technology, 2020.

[12] Sheth T N , Choudhry N K, Bowes M, et al. The Relation of Conjunctival Pallor to the Presence of Anemia[J]. Journal of General Internal Medicine, 1997, 12(2):102-106.

[13] Chalco J P , Huicho L , Alamo C, et al. Accuracy of clinical pallor in the diagnosis of anaemia in children: a meta-analysis[J]. BMC Pediatrics,5,1(2005-12-08), 2005, 5(1):46.

[14] Mitani A , Huang A, Venugopalan S , et al. Detection of anaemia from retinal fundus images via deep learning[J]. Nature Biomedical Engineering, 2020, 4(Suppl.):1-10.

[15] Wang E J , Li W , Hawkins D , et al. HemaApp: noninvasive blood screening of hemoglobin using smartphone cameras.[J]. GetMobile Mobile Computing and Communications, 2017, 21(2):26-30.

[16] Jain P , Bauskar S, Gyanchandani M . Neural network based non-invasive method to detect anemia from images of eye conjunctiva[J]. International Journal of Imaging Systems and Technology, 2020.

[17] Kasivisw An Ath An S, Vijayan T B , Simone L, et al. Semantic Segmentation of Conjunctiva Region for Non-Invasive Anemia Detection Applications[J]. Electronics, 2020, 9(8):1309.

[18] Du J, et a. An Overview of Multi-Modal Medical Image Fusion. Neurocomputing, 2016, 215: $3-20$.

[19] Zhou T, Ruan S, Canu S. A review: Deep learning for medical image segmentation using multi-modality fusion. Array, 2019, 3-4: 100004.

[20] Dolz J, Ayed I B, Jing Y, et al. HyperDense-Net: A hyper-densely connected CNN for multi- 
modal image segmentation. IEEE Transactions on Medical Imaging, 2019, 38(5): 1116-1126.

[21] Kumar A, Fulham M, Fen D, Kim J. Co-Learning Feature Fusion Maps From PET-CT Images of Lung Cancer. IEEE Transactions on Medical Imaging, 2020, 39, 204-217.

[22] Qian X, et al. Prospective assessment of breast cancer risk from multimodal multiview ultrasound images via clinically applicable deep learning. Nature biomedical engineering. 2021.

[23] Chen H, et al., MMFNet: A multi-modality MRI fusion network for segmentation of nasopharyngeal carcinoma, Neurocomputing., 2020(394):27-40.

[24] Lassau N, et al. Integrating deep learning CT-scan model, biological and clinical variables to predict severity of COVID-19 patients. Nature Communications2021, 12:634.

[25] Tan M , Le Q V . EfficientNet: Rethinking Model Scaling for Convolutional Neural Networks. 2019. Proceedings of the 36th International Conference on Machine Learning, 2019

[26] Sandler M , Howard A, Zhu M , et al. MobileNetV2: Inverted Residuals and Linear Bottlenecks[C]// 2018 IEEE/CVF Conference on Computer Vision and Pattern Recognition (CVPR). IEEE, 2018.

[27] Tan M , Chen B , Pang R, et al. MnasNet: Platform-Aware Neural Architecture Search for Mobile[C]// 2019 IEEE/CVF Conference on Computer Vision and Pattern Recognition (CVPR). IEEE, 2019.

[28] Lin T, Goyal P, Girshick R, et al. Focal Loss for Dense Object Detection[C]// 2017 IEEE International Conference on Computer Vision (ICCV). IEEE, 2017:2999-3007. 